\title{
Management of Extrahepatic Manifestations in Alcoholic Liver Disease
}

\author{
Joaquim Fernández-Solà, M.D., Ph.D.
}

Alcoholic liver disease (ALD) is often accompanied by a wide variety of extrahepatic manifestations. ${ }^{1,2}$ The World Health Organization report (2006) on excessive ethanol (EtOH) consumption identified more than 60 alcohol-related diseases. ${ }^{3}$ The systemic effects of $\mathrm{EtOH}$ include damage to the digestive tract, the central and peripheral nervous systems, the heart and vascular system, and bone and skeletal muscle; disruption of nutritional status; and damage to the endocrine and immune systems. In addition, alcohol consumption increases the risk of cancer (Table 1). There is a positive correlation between extrahepatic manifestations such as alcoholic cardiomyopathy and the presence of cirrhosis. ${ }^{4}$ Indeed, the greater the liver damage caused by $\mathrm{EtOH}$, the greater the likelihood of systemic effects existing in the same patient.

\section{Why Is EtOH Different from Other Ingested Molecules?}

The EtOH molecule displays extraordinary interactive biological reactivity. ${ }^{5}$ Its hydrophilic and lipophilic properties confer a global body diffusion potential, reaching subcellular compartments. EtOH is a potent enzymatic inducer, and its metabolites (acetaldehyde-acetate, fatty acid $\mathrm{EtOH}$ esters, EtOH-protein adducts) are very active, causing oxidative and inflammatory damage. ${ }^{6}$ EtOH interferes with cell signaling inducing cell apoptosis, decreases cell regeneration processes with impairment of extracellular matrix turnover, disrupts protein synthesis, and promotes tissue fibrosis. It is also a sensitizing agent that easily interferes with cell structures (membranes, channels, receptors) and interacts with proteins, thereby generating adducts and immunocomplexes, and disturbs hormonal, metabolic, energetic, and growth factor effects (ghrelin, leptin, insulin-like growth factor-1, and myostatin).

\section{Why Does EtOH Affect Some Patients More than Others?}

Each individual carries inherent propensities for and protection against end-organ damage due to alcohol consumption. We are beginning to learn about many polymorphisms, both in alcohol metabolic pathways and in other pathways not previously recognized as influenced by alcohol or its metabolytes, that confer different levels of risk for alcohol- associated damage to specific organs. In addition, women are more susceptible than men to develop alcoholrelated organ damage at similar levels of alcohol consumption. Another area of expanding knowledge concerns organspecific factors that confer susceptibility to alcohol-mediated damage. These factors may be specific to the type of cell (epithelial, metabolic active, excitable, embrionic), enzymatic or metabolic factors, or nonspecific factors (genetic predisposition, sex).

\section{Evaluation for Extrahepatic Injury and Clinical Planning in Patients with ALD}

The recognition of excessive use of alcohol and its harmful effects on tissues requires clinical suspicion. Clinical assessment starts with a proper alcohol history, which quantifies EtOH consumption (acute, recent, and cumulated doses). A history of significant alcohol consumption should trigger a consideration of potential systemic organ damage. Many patients with ALD have current or past comorbid addictions, such as cigarette addiction, which predisposes them to chronic pulmonary disease, pneumonia, pancreatitis, and cancer of the aerodigestive tracts ${ }^{7}$ or intravenous drug abuse with attendant risk of chronic viral infection. In general, progressive alcohol-induced tissue damage may be found in any specific tissue. The stages of tissue damage due to alcohol

Abbreviations: ALD, alcoholic liver disease; $E t O H$, ethanol; RAA, renin-angiotensin-aldosterone.

From the Alcohol Research Unit, Hospital Clinic, IDIPABPS, University of Barcelona, Barcelona, Spain

Potential conflict of interest: Nothing to report.

View this article online at wileyonlinelibrary.com

(C) 2013 by the American Association for the Study of Liver Diseases

doi: 10.1002/cld.167 
TABLE 1: Systemic Damage Produced by Chronic Excessive Alcohol Consumption that Should be Considered in Subjects with ALD

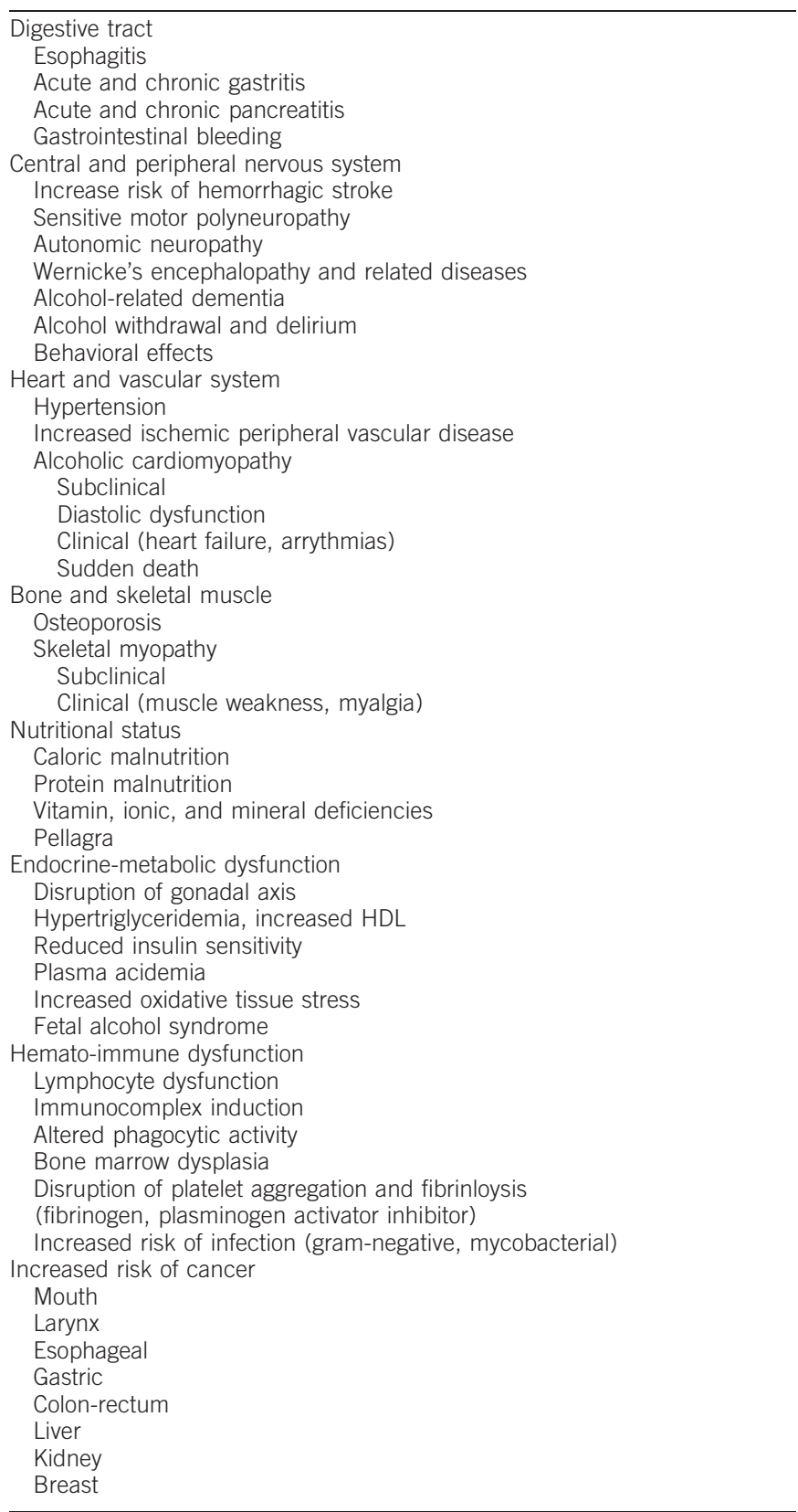

include (1) nondetectable effect; (2) transitory effect; (3) subclinical dysfunction, initially without and later with clinical damage; (4) clinical effects with reversible structural damage; and, finally, (5) advanced clinical effects with irreversible structural damage. This process may be partially reversible, albeit not in advanced or end-stage disease. In the management of ALD patients, the clinician should know the state of damage in each organ potentially involved. The final element in the clinical assessment involves incorporating the extrahepatic tissues into the global treatment plan. The goal should be to treat all comorbidities coexisting with liver damage.

\section{Treatment of Extrahepatic End-Organ Injury in Patients with ALD}

All treatment regimens start with alcohol cessation. This sine qua non will not be repeated here. In addition, we propose 10 main objectives.

1. Neurological damage in alcoholism is related to the direct effects of EtOH on neural tissue, combined with thiamine deficiency and malnutrition. Brain damage may be clinically suspected by cognitive impairment, and confirmed by computed tomography, nuclear magnetic resonance, and cognitive tests. Peripheral neuropathy is suspected when there is distal weakness or dysesthesia and a decrease in osteo-tendinous reflexes and is confirmed via electromyography. Skeletal myopathy is suspected when there is proximal weakness, pain, and muscle atrophy and an increase in muscle enzymes (elevated creatine-kinase) and is confirmed via electromyography and muscle biopsy. These processes may respond to treatment, sometimes with prolonged supplementation with thiamine, cobalamine, pyridoxine, and folate in addition to physiotherapy. ${ }^{8}$

$\mathrm{EtOH}$ abstinence-associated delirium requires prevention and treatment with benzodiazepines or clomethiazole. Alcohol withdrawal syndrome, which, as its name implies, arises soon after stopping drinking, varies from a mild disorder of autonomic hyperactivity to a severe lifethreatening medical condition manifested by systemic hypertension, tachycardia, tremors, hyperreflexia, irritability, anxiety, headache, nausea, and vomiting. These symptoms may progress to delirium tremens, seizures, coma, cardiac arrest, and death. The recent European Association for the Study of the Liver guidelines state that "In patients with acute withdrawal syndrome and ALD, benzodiazepines are the treatment of choice" (Recommendation Al). ${ }^{9}$ After recovery from seizures ("rum fits"), maintenance antiepileptic drugs are not required.

2. Cardiovascular damage may induce a diverse array of acute or chronic cardiac arrythmias (atrial fibrillation, ventricular tachycardia, and extrasystolic). Development of heart failure in alcoholic cardiomyopathy is dose-dependent. It is manifested by exertional dyspnea, orthopnea, and peripheral edema. Chest X-ray and heart echosonograpy are the principal diagnostic procedures. Alcoholic cardiomyopathy should be managed with diuretic and renin-angiotensin-aldosterone (RAA) inhibitors as in idiopathic cardiomyopathy. ${ }^{10}$ Alcohol-related arterial hypertension is dose-dependent and usually improves with abstinence and vasodilators (RAA inhibitors, $\beta$ or $\alpha_{1}$ adrenergic blockers). Heart transplantation is only considered in long-term abstainers from alcohol. Antithrombotic treatment should be undertaken after consideration of the risks and benefits.

3. Caloric and protein malnutrition are common in chronic abusers of alcohol, ${ }^{11}$ interfering with immunity and increasing systemic organ damage. Malnutrition has a 
multifactorial etiology: poor diet, malabsorption, and protein and carbohydrate disturbances. Caloric malnutrition should be approached by anthropometric measurements (body mass index $<18 \mathrm{~kg} / \mathrm{cm}^{2}$, decrease in tricipital skinfold and lean body mass). Protein malnutrition should be suspected when there are significant reductions in serum proteins and hematology parameters (total protein, albumin, pre-albumin, retinol-binding protein, transferrin, total lymphocyte count, and hemoglobin). Malnutrition may be corrected with dietary or parenteral supplementation. Vitamin (folate, E, $B_{1}, B_{3}, B_{6}$ ) and mineral deficiencies $(\mathrm{Mg}, \mathrm{P})$ and excess $(\mathrm{Fe}, \mathrm{Cu})$ are frequently seen. Chronic pancreatitis may cause malabsorption. Pancreatic insufficiency may be suspected in the presence of steatorrhea and weight loss and may be corrected with diet and pancreatic enzyme supply. Chronic abdominal pain syndromes attributed to alcoholic pancreatitis are often intractable despite pancreatic enzyme replacement.

4. Oxidative tissue damage may be corrected with antioxidant therapy (tocopherol, selenium, carotens), although there is little evidence of their beneficial effect. Skeletal myopathy and osteopenia should be considered and treated with protein, calcium, and vitamin D supplementation and active physiotherapy. ${ }^{12}$

5. Patients with ALD are more susceptible to pneumonia (especially smokers), soft tissue infection, and sepsis in the clinical setting, indicating predisposition to gram-negative bacilli, anaerobes, mycobacteria, fungi, and listeria.

6. Management of alcoholic patients in the intensive care unit requires specific considerations, mainly concerning septic shock, drugs administered, anesthesia, and perioperative management. Patients with the most severe forms of alcohol withdrawal syndrome may require intensive care, including bronchial intubation and assisted ventilation.

7. The development of cancer (ear, nose, and throat; oropharynx; esophagus; liver; colon; breast) should be considered in any alcoholic subject as a possible additional problem to ALD. As mentioned above, many of these cancers are also related to the high correlation between cigarette smoking and ALD, ${ }^{7}$ and stopping smoking is a key element in the treatment plan of any patient with ALD who also smokes.

8. The astute practitioner will consider the potential for $\mathrm{EtOH}$ to interact with prescribed medications. Chronic ingestion of excessive amounts of alcohol may lead to induction of the metabolizing enzyme CYP2E1 with consequences for adrenergic, analgesic, anesthetic, antibiotic, and antiepileptic drugs.

9. Finally, EtOH may interfere with and increase the effects of other pathogenic agents such as hepatitis C virus, tobacco, and cocaine. In this setting, $\mathrm{EtOH}$ increases the final tissue damaging effect.

10. Future treatments should consider the use of antiapoptotic factors, specific antioxidants, and growth factors (insulinlike growth factor-1, ghrelin, myostatin inhibitors) to decrease $\mathrm{EtOH}$ - mediated organ damage and favor tissue regeneration. ${ }^{13}$

\section{Conclusion}

ALD should not be considered as limited to the liver, but as true systemic disease developing within a multisystemic scenario. In the management of extrahepatic manifestations in ALD, we should first assess the amount of alcohol consumed, determine the potential systemic damage, and thereafter prepare to treat all the active diseases that coexist with liver damage, including the central and peripheral nervous system, the heart and vascular system, bone and skeletal muscle, nutritional status, and endocrine and immune dysfunctions. A multidisciplinary approach is therefore necessary, evaluating the risk of systemic organ damage in each case.

\section{CORRESPONDENCE:}

Joaquim Fernández-Solà, M.D., Ph.D., Alcohol Research Unit, Hospital Clinic, IDIPABPS, University of Barcelona, Villarroel 170, 08036,

Barcelona, Spain. E-mail: jfernand@clinic.ub.es. 